\title{
REGIONAL VARIATIONS IN WATER LOSS FROM THE SKIN OF DISEASED SUBJECTS LIVING IN A SUBTROPICAL CLIMATE ${ }^{1}$
}

\author{
By W. A. SODEMAN AND G. E. BURCH \\ (From the Departments of Preventive Medicine and Medicine, School of Medicine, Tulane \\ University and Charity Hospital of Louisiana, New Orleans)
}

(Received for publication April 15, 1943)

Clinical examination by usual means indicates variations in the rate of sweating from the skin surfaces of various portions of the body. Attempts to measure these differences quantitatively (Kuno (1), Galeotti and Macri (2)) have confirmed these clinical observations, and the development of more accurate methods (3) has permitted a quantitative evaluation of differences from normal, in a variety of conditions which clinically suggest such changes. Such observations constitute the subject of this report.

\section{METHODS AND MATERIALS}

The measurements were made under controlled conditions in an air-conditioned room, maintained at $75^{\circ} \mathrm{F}$. $\pm 1^{\circ}$ and at a relative humidity of 50 per cent \pm 2 per cent. A change from this comfortable environment to hot and humid conditions $\left(105^{\circ} \mathrm{F} . \pm 2^{\circ}\right.$ and 75 per cent \pm 2 per cent, respectively) was produced in order to stimulate visible sweating. There were no perceptible currents of air, and, by room design, psychic disturbances were reduced to a minimum.

The technic for measurement of water loss was that used previously (4) in normal individuals. It consists, essentially, of passing dry oxygen through a closed system over a measured area of skin. The quantity of the vaporized water, trapped in aluminum coils by freezing, was calculated by the difference in the weight of the coils before and after the condensation of the water.

Studies were conducted as follows: The subject entered the observation room, where the atmosphere had previously been adjusted to a temperature of $75^{\circ} \mathrm{F}$. and a relative humidity of 50 per cent. The patient removed all of his clothing except his underwear, entered a comfortable bed with an innerspring mattress, and covered himself with cotton sheets or a woolen blanket, to suit his comfort. The collecting chambers were sealed in place, and, after a period of approximately 45 minutes, the water loss from the enclosed areas of skin was measured. The water was collected continuously throughout the entire period of study. By the manipulation of stopcocks controlling the flow of the water-laden oxygen and diverting it into one collecting coil or another, the water loss could be separated into 15-minute samples. Thus, collections of sweat for 15-minute periods were made,

1 Aided by a grant from the Rockefeller Foundation. and then, without the subject's knowledge, the thermostat and humidistat were readjusted, and the room temperature and relative humidity increased to $105^{\circ} \mathrm{F}$. and 75 per cent, respectively. About 15 minutes were required for the atmospheric conditions of the room to reach these new levels. During the period of change, and for 30 minutes thereafter, the water loss from the areas of skin under observation was measured so that two 15minute collections were made while the room was hot and humid. Duplicate or triplicate measurements were made for each part studied.

The rate of water loss from the skin was measured during the winter as well as spring and summer months ${ }^{2}$ in New Orleans. The conditions in the room were the same during all seasonal periods. We have previously shown that there is no appreciable difference in the rate of sensible or insensible water loss in these seasons under the laboratory conditions (3). Subjects were used as they became available, regardless of season.

No attempt was made to have the subjects in a postabsorptive state during the determinations. They were advised to eat a light breakfast or lunch, and were then studied about 2 to 3 hours later.

Observations were made on 14 adults displaying a variety of states enumerated below. Eleven were white, and 3 negro. There were 8 females and 6 males, varying in ages from 17 to 56 years.

The areas of skin studied were those over the right and left index finger tips, right and left second toe tips, right forearm (volar surface), anterior surface of chest, epigastrium, umbilical area, anterior surface of the right thigh, right pretibial area, right cheek, forehead, right axilla, left axilla, right leg, plantar surface of the heels of the right and left feet, and the palms of the right and left hands. Three or 4 areas were studied simultaneously.

Comparisons were made with the values obtained for normal adults (3).

\section{RESULTS}

In all, 481 separate 15-minute determinations were made on the 14 subjects. In the data, the values given are converted to milligrams per 10 square centimeters of skin area per 15 minutes.

2 Average temperature and relative humidity for New Orleans for period of January to March, inclusive, were $55.1^{\circ} \mathrm{F}$. and 68.6 per cent, respectively, and for July and August were $83.0^{\circ} \mathrm{F}$. and 80.3 per cent. 


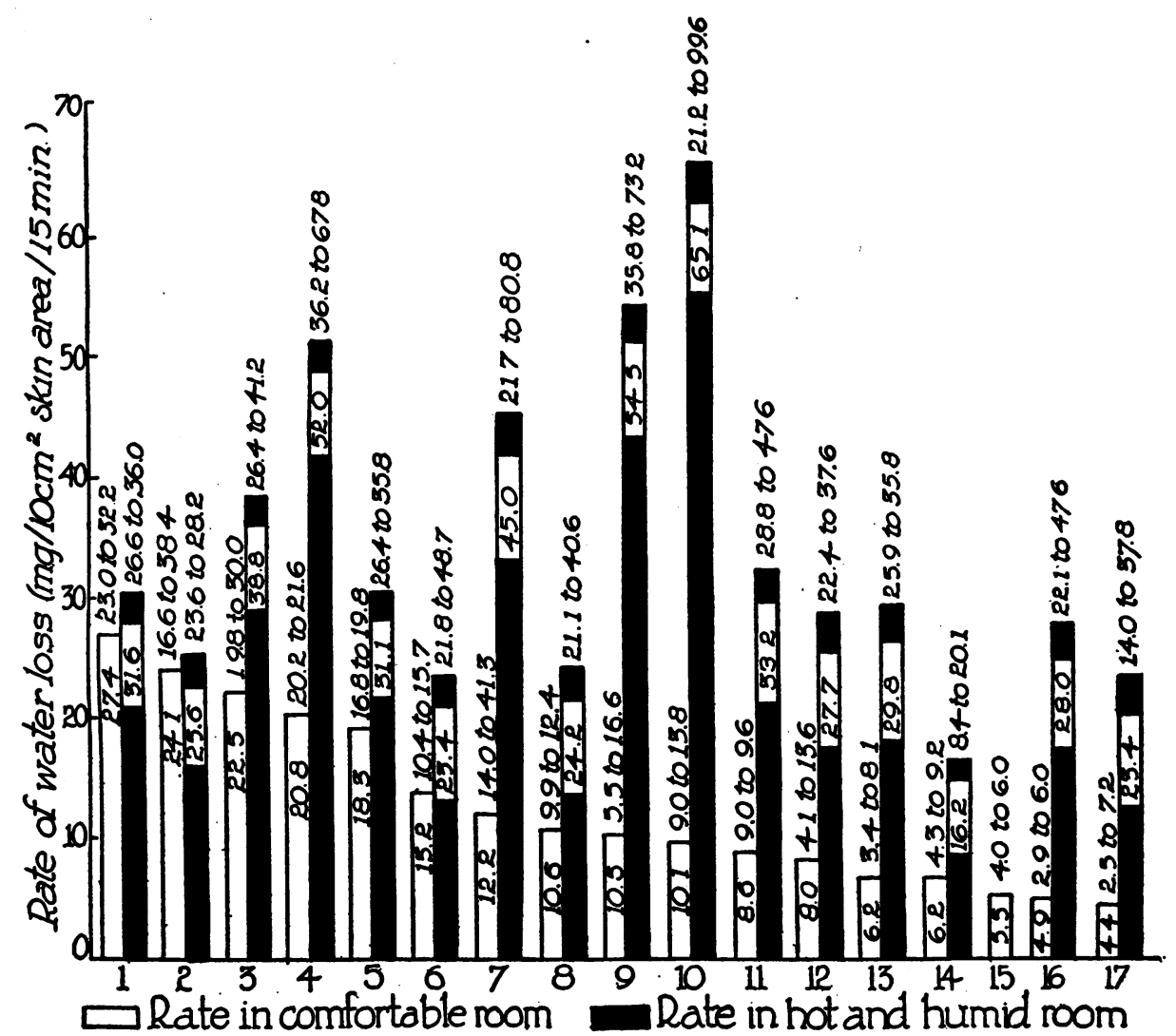

Fig. 1. The Mean and Extreme Values for the Rate of Water Loss from 17 Different Skin Areas of 37 Normal Adults Obtained During the Months of January THROUGH MARCH

The parts studied are represented as follows by the numbers along the abscissa: $1=$ Ball of the right foot, $2=$ plantar surface of the heel of the right foot, $3=$ palm of the right hand, $4=$ lateral surface of the right arm, 5= mid-plantar area of the right foot, $6=$ right second toe tip, $7=$ right index finger tip, $8=$ right cheek, $9=$ middle of forehead, $10=$ left axilla, $11=$ right axilla, $12=$ posterior surface of the right leg, $13=$ right flank, $14=$ anterior surface of the right thigh, $15=$ mid-epigastrium, $16=$ posterior surface of the left thigh, $17=$ volar surface of right forearm.

Figures 1 and 2 show the results of studies previously reported in 37 normal adults. Mean and extreme values are shown. It was noted that water loss was found to vary markedly from area to area, from patient to patient, and from time to time in the same patient (3).

Figures 3 through 7 compare the results in normal individuals with those in certain abnormal states. In Figure 3, values obtained in a patient with hypothyroidism are shown. In the comfortable room, water loss from the right index finger is below, from the right forearm is above, and from the forehead lies within the extremes found in normal individuals. In a hot humid environ- ment, values for water loss from index finger and forehead failed to rise into the range of normal, while values for the right forearm did rise into this range, although remaining below the average for that area. In another patient with hypothyroidism, values for the comfortable and hot rooms were, respectively, 13 and 19 for the right index finger; 10 and 16 for the right forearm. These values compare favorably with those of the patient in Figure 3. The right palm changed from 16 to 21 , as compared with the mean normal of 23 and 39 . Both figures show values below the minimum values found in normal subjects (Figure 1). 


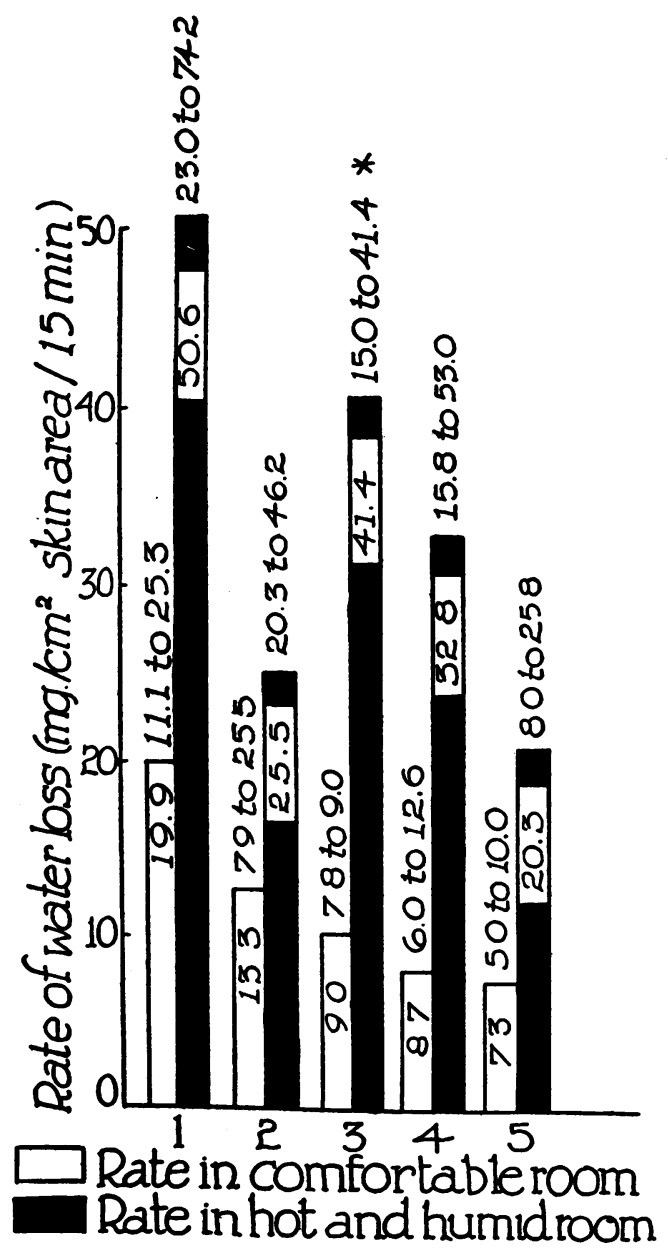

Fig. 2. The Mean and Extreme Values for the Rate of Water Loss from 5 Skin Areas of 12 Normal Adults, Obtained During the Months of July AND August

* = variation in only one patient. The parts studied are represented by numbers along the abscissa : $1=$ Right index finger tip, $2=$ right second toe tip, $3=$ middle of forehead, $4=$ volar surface of right forearm, $5=$ midepigastrium.

Figure 4 shows values for a man who claimed to suffer from summer weather. $\mathrm{He}$ stated that he could not work in hot weather without fatigue, although the same work in the same weather did not affect others. Nothing about his clinical examination indicated the cause for this complaint. Observations on him showed values for water loss in a comfortable room consistently below the normal average, and these differences were markedly accentuated in a hot, humid room, each value for the patient lying below the extreme range found in normal subjects.

Figure 5 shows the water loss for various areas in 3 patients with congenital ectodermal dysplasia. Often sweating is said to be lacking in these patients because of a disturbance in the sweat glands. In the present patients, values are seen to parallel closely the data for normal subjects, especially in the hot and humid environment.

Figure 6 represents data upon a patient with "nervous" sweating. The marked sweating of the hands in such patients, noted clinically, is quantitatively confirmed, while in the forearm and thigh the deviations from normal are slight and variable.

In Figure 7, values for a patient with scleroderma are graphically represented. Here, values in the right index finger and forehead in the comfortable and in the hot rooms were below the range of normal subjects. In the comfortable room, values in the right forearm and epigastrium were in the normal range. In the hot room, the forearm reacted as did the finger and forehead. Normal values for the epigastrium were not available for comparison. Most marked differences correspond to the areas of major involvement

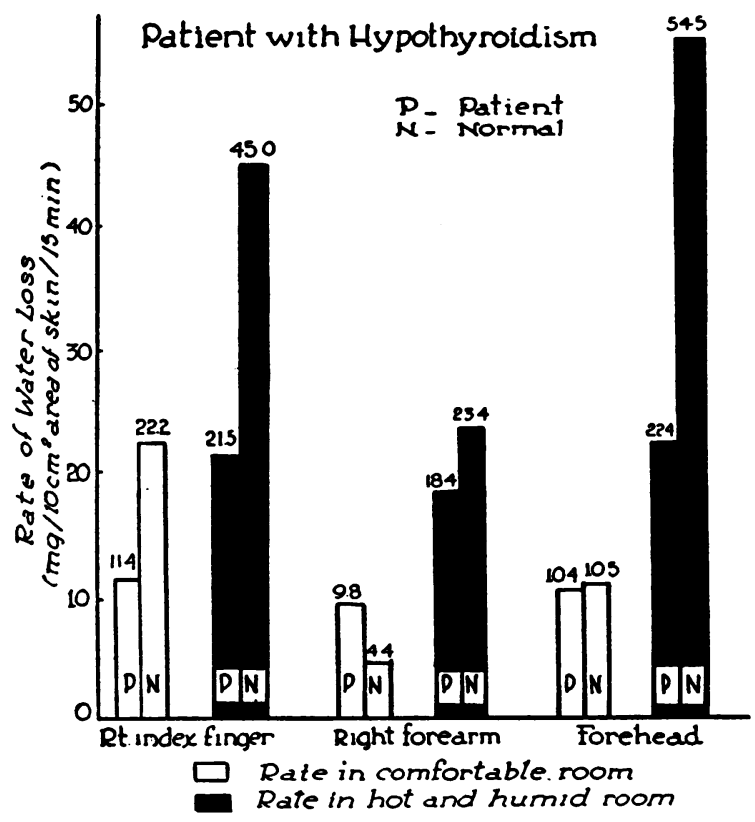

Fig. 3. The Rate of Water Loss from the Skin Surfaces of 3 Areas of a Patient Suffering with HYPOTHYROIDISM 


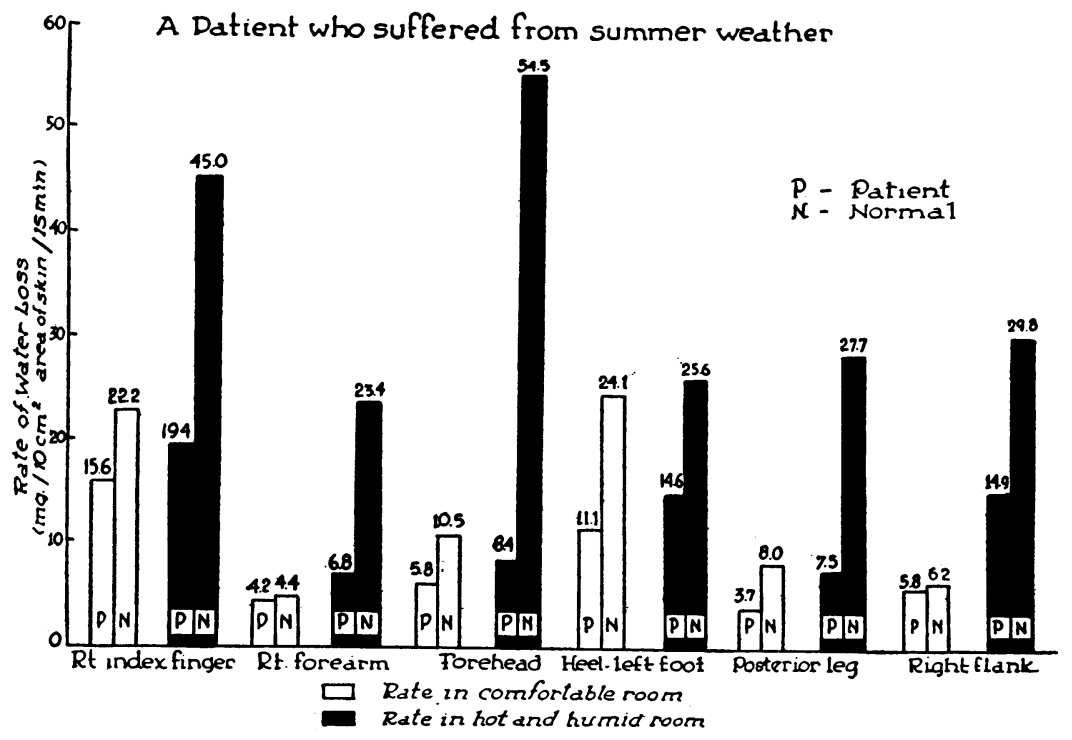

Fig. 4. The Rate of Water Loss from the Skin of Several Areas of the Body of a Subject Who Suffered Intensely in a Hot Climate

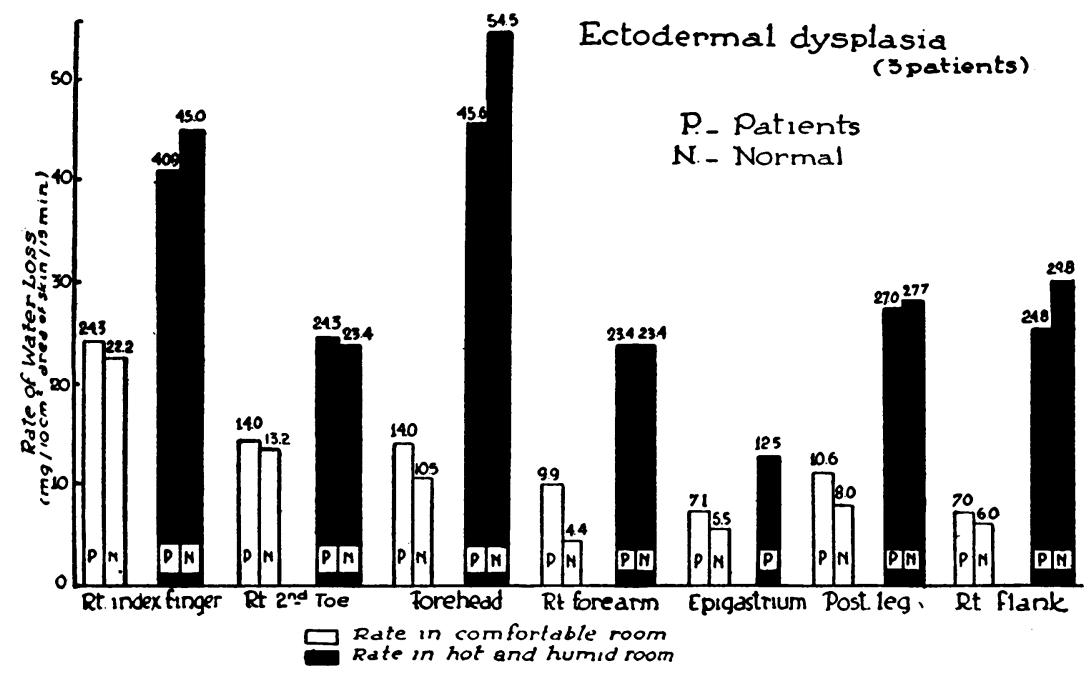

Fig. 5. The Mean Rates of Water Loss from the Skin of Several Areas of 3 Adult Members of a Family Who Had Congenital Ectodermal Dysplasia

with the sclerodermatous process. In another patient with early scleroderma and Raynaud's disease, values before and after elevation of room temperature, respectively, were for the following areas, 13 and 61 for the right index finger, 11 and 72 for the right forearm, 13 and 79 for the right pretibial area. The marked variations from normal, seen in the previous patient, are not evident here. In a patient with Raynaud's disease with scleroderma, the values were 23 and 40, 17 and 36 for the right and left index fingers, respectively, and 10 and 33 for the right leg. Thus, the figures for this patient fall within the normal range.

A patient with gigantism, acromegaly, and diabetes mellitus complained of dripping wet hands. Her blood Wassermann reaction was positive and her basal metabolic rate, -2 per cent. Values for her right index finger exceeded normal in both environments. They were, respectively, 73 and 


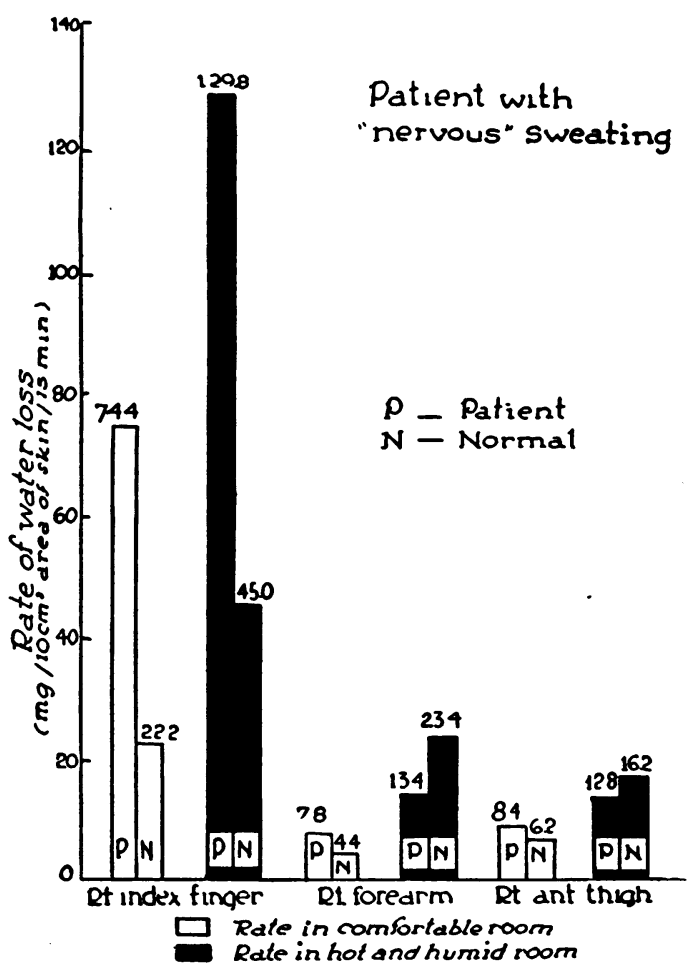

Fig. 6. The Rate of Water Loss from the Skin of Several Areas of the Body of a Subject Who Was SUfFering With NeURoctrculatory Asthenia aND "Nervous" Sweating
83. Both the right and left axillae showed high values, 20 and 18, in a comfortable room, but values in normal range, 40 and 42 in the hot environment. The forearm and forehead showed similar changes, 8 to 18 , and 17 to 35 , respectively. In the cheek, both values, 8 and 13 , were lower than normal.

A patient with Buerger's disease displayed normal values, 15 to 22 in the right index finger, similar but lower results, 9 and 20, in the second right toe, which, when repeated on another day, were distinctly low, 8 to 10 . The values for the forehead fell in normal range, 16 to 71 . In the umbilical area, the figures were 7 and 17 .

A patient with syringomyelia, studied only in a comfortable room, showed no change from normal. Right index finger, right forearm, and forehead values, were 21,5 , and 12 , respectively.

The effects on water loss of a weeping allergic eczema, due to wheat sensitization in a baker, were studied. Values were, for the right palm, 16 and 16 ; right side of chest, 11 and 12 ; right index finger, 8 and 25 ; left heel, 15 and 23 ; and right heel, 17 and 24 . The low initial values in the comfortable room and the failure to develop of the marked rises expected in the hot humid room are evident.

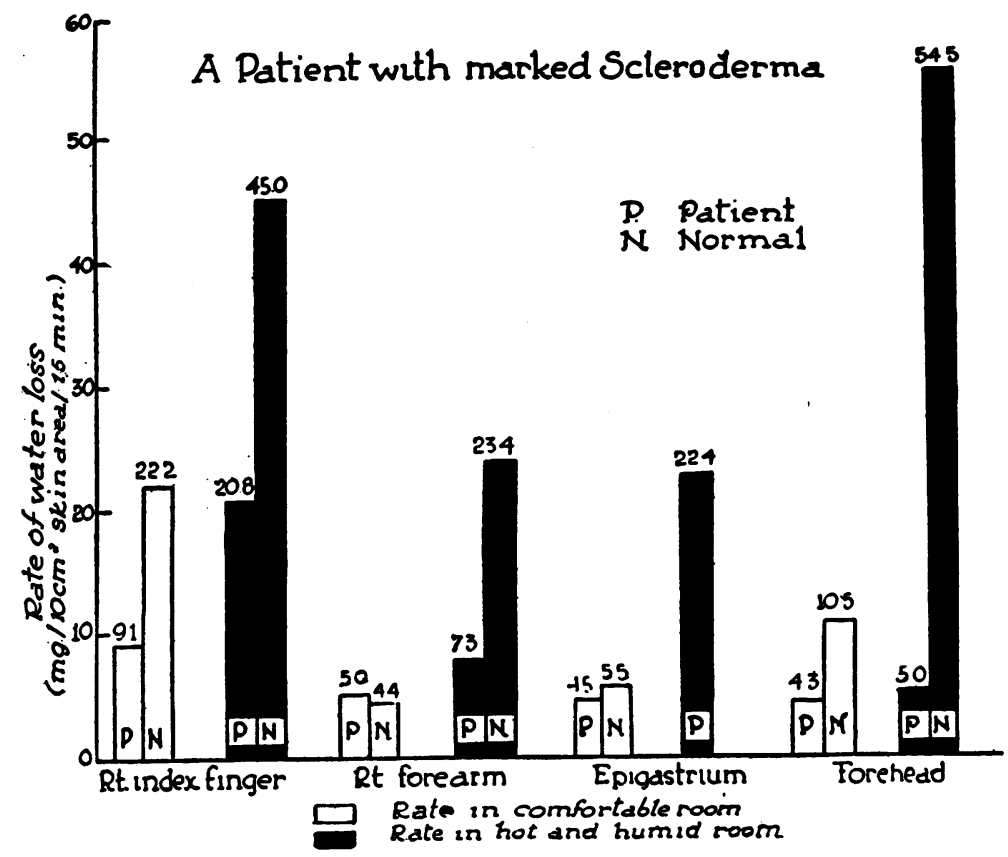

Fig. 7. The Rate of Water Loss from the Skin of Several Areas of the Body of a Subject Suffering with Scleroderma 


\section{DISCUSSION}

The marked variations in water loss from area to area, from patient to patient, and from time to time in the same patient, must be emphasized. In normal subjects, the variations followed, in general, a definite pattern (3). In descending order, the values for water loss are hands, feet, head, arms, legs, and trunk as seen in Figure 1. In the hot and humid environment, the rate was greatest for the finger tip, axillae, and forehead. It was also noted in the study of normal individuals that the percentage increase, due to transition from the comfortable to the hot room, was as great, or greater, from the skin of the arms, legs, and trunk, as from the finger and toe tips.

Despite the variations in normal individuals from area to area and from person to person, there is evident, as stated above, a general plan or trend of values in the whole group.

The reaction of the thermal stimulus involved all areas studied and presumably involves the entire body. By this means, the organism regulates heat loss as radiation becomes less and less important. Finally, when environmental temperature exceeds body temperature, evaporation becomes the sole means of heat regulation. Variations from normal in this thermal response are to be predicted in hypothyroidism, for in this condition, heat exchange is below normal.

In the patient with the complaint of discomfort in warm weather, there was no obvious evidence to substantiate this complaint until quantitative sweat studies were done and clearly indicated a marked inability to sweat normally with rising environmental temperature. No doubt, other such patients exist. Such physiologic states may account for marked discomfort and inefficiency in men living in subtropical and tropical climates. The abnormal response is not yet explained. Both neurogenic and circulatory control has been postulated to explain the thermal response, but we have not yet been able to demonstrate the importance of either mechanism in this patient.

The disturbed responses in the patients with allergic eczema and scleroderma indicate the possible influence of local dermal factors on water exchange. One would expect that interference with water exchange by local dermal factors might increase water loss through uninvolved, or less involved areas. The values for the patient with scleroderma suggest this possibility. Under any circumstances, these patients demonstrate the effect of local factors on sweating.

Reactions of the sweating mechanism are shown to vary with the stimulus. The general response to the thermal stimulus has just been discussed. Emotional stimuli evoke a different response, increasing the sweat production especially in the hands. This is evident clinically, and our patient with "nervous" sweating obviously falls into this group. In the right index finger in the comfortable room, the values exceeded those of the normal in a hot humid room. The response to heat was excessive, but similar, on a percentage basis, to that of the normal. These results did not hold in the other areas studied. Patients of this type demonstrate regional variations in sweating unlike those in the patients with scleroderma and eczema described above, and resting upon disturbances in the nervous control of the sweating mechanism.

The differences in the response to heat of the finger and of the forearm and the thigh, as compared to each other as well as to the results in normal subjects, would seem to favor the predominance of non-nervous factors in the thermal response in general. However, the group of patients herein studied is so heterogeneous that a series of each type will be necessary before such general conclusions may be drawn.

\section{SUM MARY}

A comparison of a group of patients with a variety of conditions affecting the sweating mechanism with a group of normal individuals indicates a much increased variation in the rate of sweating, both insensible and sensible. The conditions vary from those affecting the general controlling factors of the sweat mechanism to local diseases of the skin which appear to interfere mechanically with the process. Obviously, local factors in the sweat glands and skin, so often neglected, may be important in sweat responses.

Variations from normal in nervous sweating and the reactions of these patients to heat-stimula- 
tion favor a non-nervous mechanism as the important factor in the thermal response.

Vascular disturbances, such as those found in Buerger's and Raynaud's diseases, which might be expected to affect appreciably the sweating mechanism, had little influence on these reactions.

Quantitative studies of sweat production have been found helpful in establishing objective evidence to account for otherwise inexplicable reactions to heat.

We wish to express our appreciation for the technical assistance and keen interest of Mr. G. Morgavi, who participated in these studies.

\section{BIBLIOGRAPHY}

1. Kuno, Y., The Physiology of Human Perspiration. J. \& A. Churchill, Ltd., London, 1934.

2. Galeotti, G., and Macri, N. M., Uber die Perspiratio insensibilis unter normalen und pathologischen Bedingungen. Biochem. Ztschr., 1914, 67, 472.

3. Burch, G. E., and Sodeman, W. A., Regional relationships of rate of water loss in normal adults in a subtropical climate. Am. J. Physiol., 1943, 138, 603.

4. Neumann, C., Cohn, A. E., and Burch, G. E., A quantitative method for the measurement of the rate of water loss from small areas, with results for finger tip, toe tip, and postero-superior portion of the pinna of normal resting adults. Am. J. Physiol., 1941, 132, 748. 